\title{
ANÁLISE DOS EFEITOS DA EXPOSIÇÃO CRÔNICA ORAL AO HERBICIDA À BASE DE GLIFOSATO NA
} REMODELAÇÃO CARDÍACA EM RATOS

\author{
Larissa Ferreira Ros, Cristiane Pontes Teodozio, Ana Julia Alias Costa, Renata Calciolari Rossi, Rejane Batista \\ Brinholi, Francis Lopes Pacagnelli
}

Universidade do Oeste Paulista - UNOESTE, Presidente Prudente, SP. E-mail: larissaf.ros@hotmail.com

\section{RESUMO}

O objetivo deste estudo foi analisar a remodelação cardíaca estrutural de ratos expostos ao herbicida glifosato de forma crônica. Foram utilizados 20 ratos Wistar adultos machos (300-450 g), divididos em grupo controle $(C T, n=10)$ e grupo de ratos alimentados com ração contaminada por glifosato $(G O, n=10)$ por 180 dias. Após o período experimental os ratos foram eutanasiados e para avaliar a remodelação cardíaca estrutural o coração foi dissecado em ventrículo esquerdo (VE), átrios (ATs) e ventrículo direito (VD) e pesados. Houve aumento dos átrios ( $C T=0,084 \pm 0,22 \mathrm{mg}$ e $\mathrm{GO}=0,105 \pm 0,21 \mathrm{mg}, \mathrm{p}=0,048)$, ventrículos direito ( $C T=0,219 \pm 0,04 \mathrm{mg}$ e $\mathrm{GO}=0,276 \pm 0,03 \mathrm{mg}, \mathrm{p}=0,036)$ e esquerdo $(\mathrm{CT}=0,950 \pm 0,05 \mathrm{mg}$ e $\mathrm{GO}=$ $1,030 \pm 0,09 \mathrm{mg}, p=0,040$ ) no grupo exposto ao glifosato. $O$ glifosato promoveu aumento das câmaras cardíacas de ratos que ingeriram ração contaminada.

Palavras-chaves: doenças cardiovasculares, herbicidas, glifosato, coração, hipertrofia

\section{ANALYSIS OF THE EFFECTS OF ORAL EXPOSURE TO THE GLYPHOSATE HERBICIDE ON CARDIAC REMODELING IN RATS}

\begin{abstract}
The aim of this study was to analyze the structural cardiac remodeling of rats exposed to the herbicide glyphosate chronically. Twenty male adult Wistar rats $(300-450 \mathrm{~g})$ were used, divided into a control group (CG, $n=10)$ and group of rats fed diet contaminated by glyphosate (OG), $n=10$ ) for 180 days. After the experimental period, the rats were euthanized and to evaluate structural cardiac remodeling the heart was dissected in the left ventricle (LV), atriums (ATs) and right ventricle (RV) and heavy. There was an increase in the atrium ( $C G=0,084 \pm 0,22 \mathrm{mg}$ e $O G 0,105 \pm 0,21 \mathrm{mg}, p=0,048)$, right ventricles $(C G=0,219 \pm 0,04 \mathrm{mg}$ e $O G=$ $0,276 \pm 0,03 \mathrm{mg}, p=0,036)$ and left ventricles ( $C G=0,950 \pm 0,05 \mathrm{mg}$ e $O G=1,030 \pm 0,09 \mathrm{mg}, p=0,040$ ) in the group exposed to glyphosate. Glyphosate promoted an increase in the cardiac chambers of rats that ingested contaminated feed.
\end{abstract}

Keywords: cardiovascular disease, herbicides, glyphosate, heart, hypertrophy

\section{INTRODUÇÃO}

O glifosato (Roundap ${ }^{\circledR}$ ) pertence a classe de herbicida não-seletivo de ação sistêmica do grupo químico glicina substituída, altamente tóxico, é composto por Sal de Di-amônio de N(phosphonomethyl) glycine, equivalente ácido de $\mathrm{N}$-(phosphonomethyl) glycine e outros ingredientes ${ }^{1}$, é recomendado para o controle pós-emergente de plantas infestantes, é solúvel em água, facilitando a contaminação do ambiente quando entra em contato com a água e $\mathrm{o}$ ar, atingindo até mesmo águas subterrâneas ${ }^{1,2}$.

Os herbicidas a base de Glifosato (GBH) são vastamente aplicados em diversas culturas de grãos e cereais como, plantações de arroz irrigado, café, cana-de-açúcar, citrus, milho, soja, granola, cevada, feijão, entre outras ${ }^{1,3}$. Entre aqueles que mais sofrem com o uso exagerado de 
agroquímicos, trabalhadores rurais ou quaisquer pessoas que trabalhem com este tipo de herbicida tem um grande risco de contaminação, podendo ocorrer por exposição dérmica, digestiva e mucosas, sem a utilização de equipamentos de proteção individual ${ }^{4}$.

A utilização do GBHs tem sido intensa desde os anos 70 , porém pouco se sabe sobre sua toxicidade, sendo poucos estudos que investigam o efeito do glifosato sobre o coração ou seu desenvolvimento ${ }^{4}$. Esse herbicida atua sobre uma enzima vegetal chave, a 5-enolpiruvilchiquimato3-fosfato sintase, não presente em vertebrados, incluindo seres humanos, acreditando-se que são espécies não-alvo, entretanto, há estudos que comprovam o oposto ${ }^{5}$.

Embora os testes iniciais realizados pela indústria química sugerissem baixo risco para as espécies não-alvo, há evidências crescentes de uma série de espécies de vertebrados, incluindo sapos, peixes, ratos e até mesmo estudos epidemiológicos humanos sugerem o contrário. Dada a provável exposição de animais e humanos pelo ar, perto de terras agrícolas, água potável contaminada e culturas alimentares, há a necessidade de um novo olhar sobre a toxicidade do glifosato ${ }^{3}$.

O objetivo deste estudo foi avaliar os efeitos da ingestão crônica do herbicida glifosato na remodelação cardíaca de ratos por meio da avaliação de parâmetros estruturais.

\section{MÉTODOS}

\section{ASPECTOS DE NATUREZA ÉTICA}

O projeto foi aprovado pelo Comitê de Ética para uso dos Animais da Universidade do Oeste Paulista (UNOESTE), protocolo 6291. Este estudo foi conduzido de acordo com as recomendações da Sociedade Brasileira de Ciência em Animais de Laboratório.

\section{CARACTERIZAÇÃO DA AMOSTRA}

Foram utilizados 20 ratos Wistar adultos machos (300-450g), fornecidos pelo Biotério Central da UNOESTE, alocados em gaiolas plásticas coletivas ( 3 animais por gaiola e uma gaiola com 2 animais) medindo $600 \times 415$ milímetros, a temperatura média de $22 \pm 2 \circ \mathrm{C}$, com ciclos de 12 horas de luminosidade, sendo das 07:00 as 19:00 horas (período claro) e 19:00 as 07:00 horas (período escuro), com umidade de ar controlada à $50 \pm 15 \%$.

\section{PROTOCOLO DE EXPOSIÇÃO ORAL AO HERBICIDA GLIFOSATO}

O protocolo de exposição contou com uma caixa $(32 \times 24 \times 32 \mathrm{~cm})$, ligada a um nebulizador ultrassônico da marca Pulmosonic Star $^{\circledast}$. As concentrações do herbicida foram administradas após serem diluídas em $10 \mathrm{ml}$ de água destilada. Ambos os grupos foram expostos por um tempo aproximado de 15 minutos diários, de segunda à sexta-feira, por um período de 180 dias.

Os animais foram divididos aleatoriamente em 2 grupos:

- Grupo controle - CT ( $n=10)$ : receberam dieta padrão nebulizada com solução contendo $10 \mathrm{ml}$ de água destilada.

- Grupo de glifosato oral - GO: $(n=10)$ : receberam dieta padrão nebulizada com o herbicida, sendo $3,71 \times 10^{-3}$ gramas de ingrediente ativo por hectare (g.i.a/há) que é equivalente a $187,17 \mathrm{mg} / \mathrm{m}^{3}$. Essa dose foi escolhida pois representa a quantidade utilizada do herbicida na agricultura e foi ajustada para o tamanho da caixa.

A dieta padrão para animais foi nebulizada (400 g) por caixa as segundas e quintas-feiras. Para se obter o controle da ração ingerida (consumo alimentar) em cada caixa, representando a média de consumo de 3 ou 2 animais a ração foi pesada as terças e sextasfeiras e $400 \mathrm{~g}$ de ração nebulizada eram novamente oferecidas.

Os animais receberam dieta padrão para animais de laboratório $\left(\right.$ Primor $\left.^{\circledR}\right)$ e água $a d$ libitum.

\section{ANÁLISE DA REMODELAÇÃO CARDÍACA ESTRUTURAL}

Após o período experimental os ratos foram pesados (PCF), eutanasiados e o coração dissecado em ventrículo esquerdo (VE), átrios (ATs) e ventrículo direito (VD) e pesados.

\section{ANÁLISE ESTATÍSTICA}

Os dados foram expressos em média \pm desvio padrão. Para avaliar a normalidade foi utilizado o teste de Shapiro Wilk, sendo considerados paramétricos, utilizou-se o teste $\mathrm{T}$ não-pareado para comparação entre os grupos. Foi utilizado o programa estatístico Graphpad Prism. O valor de $\mathrm{p}<0,05$ foi considerado estatisticamente significativo. 


\section{RESULTADOS}

Não houve alteração do peso corporal final entre os grupos avaliados ( $C T=462,8 \pm 35,43$ $\mathrm{mg}$ e $\mathrm{GO}=443,3 \pm 33,13 \mathrm{mg}$ ). Os átrios (ATs) obtiveram aumento no grupo exposto em relação ao grupo controle ( $\mathrm{CT}=0,084 \pm 0,22 \mathrm{mg}$ e $\mathrm{GO}=$ $0,105 \pm 0,21 \mathrm{mg}, \quad p=0,048)$, assim como no ventrículo direito (VD) $(C T=0,219 \pm 0,04 \mathrm{mg}$ e $\mathrm{GO}=$ $0,276 \pm 0,03 \mathrm{mg}, \quad p=0,036)$ e no ventrículo esquerdo (VE) (CT=0,950 $\pm 0,05 \mathrm{mg}$ e $\mathrm{GO}=$ $1,030 \pm 0,09 \mathrm{mg}, p=0,040$ ) (Figura 1)

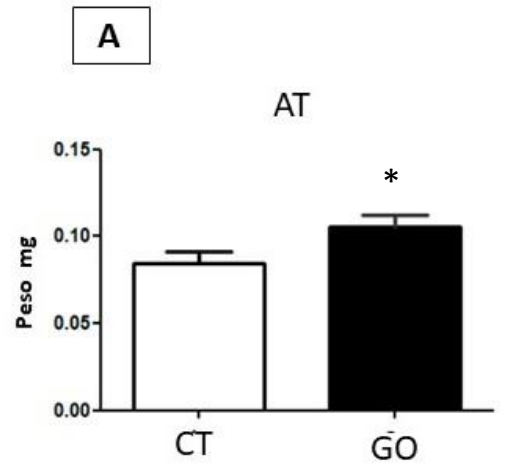

\section{B}

VD

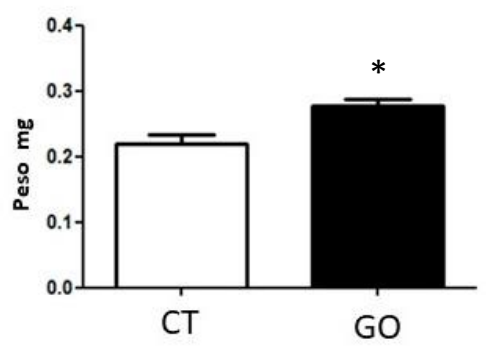

C VE

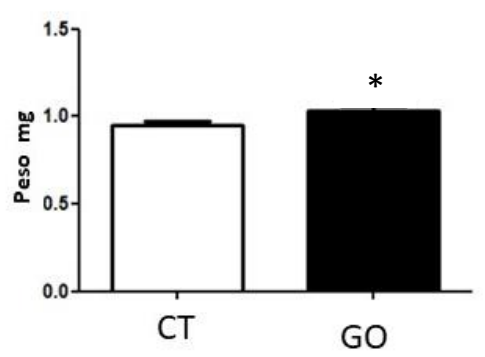

Figura 1. A. Peso dos átrios (AT) B. Peso do ventrículo direito (VD) C. Peso do ventrículo esquerdo (VE); CT= Grupo controle. $\mathrm{GO}=$ Grupo glifosato oral. Teste T não-pareado, ${ }^{*} p<0,05$ vs. CT.

\section{DISCUSSÃO}

O principal achado desse estudo foi que o uso do glifosato oral de forma crônica promoveu aumento das câmaras cardíacas, sendo isso um indicativo de remodelação cardíaca estrutural.

Os herbicidas a base de glifosato (GBHs) são vastamente aplicados em diversas culturas de grãos e cereais, e a sua utilização tem sido intensa desde os anos 70, para aumentar a produtividade ${ }^{4}$. Testes realizados por agências regulatórias, e alguns estudos, sugeriram que a exposição direta teria toxicidade mínima ou nenhuma, ou seja, a exposição ao glifosato não traria riscos para as espécies não-alvo. Mas, estudo mais recente mostra que a exposição ao herbicida traz sim riscos devido a sua toxicidade, e diante disso há a necessidade de estudar mais profundamente os danos que que esse herbicida pode ocasionar ${ }^{3,7}$.

Outros herbicidas vêm sendo estudados em relação aos seus efeitos cardiotóxicos. O herbicida 2,4D inalado agudamente promoveu hipertrofia de cardiomiócitos em camundongos, mostrando seu potencial em causar remodelamento cardíaco patológico ${ }^{6}$.

Um estudo sobre a toxicidade do glifosato no sistema cardiovascular de Zebrafish monitorou a diferenciação precoce dos cardiomiócitos. A exposição ao glifosato durante o desenvolvimento do coração, diminuiu a frequência cardíaca, induziu a anomalias e alterações vasculares cardíacas, demonstrando a toxicidade desse herbicida ${ }^{2}$. 
Alguns estudos tem demonstrado a toxicidade ocasionada pelo glifosato em outros órgãos. A utilização de baixas doses e de forma crônica ocasionou disfunção hepática demonstrada por doença hepática não alcoólica e progressão para esteatose ${ }^{8}$. Foi demonstrado também que ocorrem epimutações dos espermatozoides contribuindo para uma alteração epigenética transgeracional ${ }^{9}$. Outro estudo demonstrou danos relacionados ao estresse oxidativo no cérebro, rins, fígado e coração de ratos submetidos a uma dose de 375 $\mathrm{mg} \mathrm{kg}^{-1}$ durante 7 semanas ${ }^{10}$.

A exposição sub crônica do glifosato ocasionou alteração da remodelação cardíaca em relação aos aspectos bioquímicos demonstrado pelo aumento da peroxidação lipídica avaliado pelo MDA, diminuição de enzimas antioxidantes avaliada pela glutationa, e aumento de biomarcadores de necrose celular $(C K M B)^{10}$. Foram observados também danos como a apoptose das células do músculo cardíaco, degeneração hialina e hemorragia cardíaca ${ }^{10}$.

Além dessas alterações já encontradas evidenciamos um aumento das câmaras cardíacas com a utilização crônica do glifosato, demonstrando seu potencial efeito tóxico cardíaco. Esse aumento do peso estrutural cardíaco pode estar relacionado a hipertrofia excêntrica dos cardiomiócitos, acúmulo de tecido conjuntivo, promovendo alterações funcionais importantes. Mais estudos que abordem outros aspectos da remodelação cardíaca são necessários com abordagem quantitativa das áreas dos cardiomiócitos, análise de tecido conjuntivo e avaliações funcionais.

\section{CONCLUSÃO}

A exposição crônica oral ao herbicida à base de glifosato promoveu alterações estruturais na remodelação cardíaca em ratos.

\section{AGRADECIMENTOS E CONFLITO DE INTERESSE}

Agradecemos a médica veterinária Gracielle Vieira Gonçalves pelo suporte dado durante a realização dessa pesquisa.

Os autores declaram não haver qualquer potencial conflito de interesse que possa interferir na imparcialidade deste trabalho científico.

\section{REFERÊNCIAS}

1. Roundup Original'DI. São Paulo. Fabricante Monsanto do Brasil LTDA. Bula disponível em:
http://www.adapar.pr.gov.br/arquivos/File/defis /DFI/Bulas/Herbicidas/rounduporiginaldi190118. pdf/.

2. Roy NM, Ochs J, Zambrzycka E, Anderson A. Glyphosate induces cardiovascular toxicity in danio rerio. Environ Toxicol Pharmacol. 2016; 46:292-300.

https://doi.org/10.1016/j.etap.2016.08.010.

3. Myers JP, Antoniou MN, Blumberg B, Carroll L, Colborn T, Everett LG, et al. Concerns over use of glyphosate-based herbicides and risks associated with exposures: a consensus statement. Environ health.

2016;15:19.

https://doi.org/10.1186/s12940-016-0117-0.

4. Mantovani RO, Pinheiro DG, Oliveira GLF, Perrud SN, Teixeira GR, Nai GA, et al. Effect of different doses of 2,4-dichlorophenoxyacetic acid $(2,4-D)$ on cardiac parameters in male Wistar rats. 2020. In Press

5. Vandenberg LN, Blumberg B, Antoniou MN, Benbrook CM, Carroll G, Colborn T, et al. Is it time to reassess current safety standards for glyphosate-based herbicides? J Epidemiol Community Health. 2017; 71(6):613-8. https://doi.org/10.1136\%2Fjech-2016-208463.

6. Negrão ALR, Oliveira BD, Gonçalves MDG, Mariano TB, Oliveira TFDS, Sabela AKDA, et al. Effect of Short-Term Inhalation of The Herbicide 2,4D on Cardiac Remodeling: Morphological Aspects. Int J Cardiovasc Sci. 2019; 32(3):247-52. https://doi.org/10.5935/2359-4802.20190014.

7. Mesnage R, Defarge N, Spiroux de Vendomois $J$, Seralini GE. Potential toxic effects of glyphosate and its commercial formulations below regulatory limits. Food Chem Toxicol. 2015; 84:133-53.

https://doi.org/10.1016/i.fct.2015.08.012.

8. Mesnage R, Renney G, Séralini GE, Ward M, Antoniou MN. Author Correction: Multiomics reveal non-alcoholic fatty liver disease in rats following chronic exposure to an ultra-low dose of Roundup herbicide. Sci Rep 8. 201812572. https://doi.org/10.1038/s41598-018-30760-8

9. Kubsad D, Nilsson EE, King SE, Sadler-

Riggleman I, Beck D, Skinner MK. Assessment of

Glyphosate Induced Epigenetic Transgenerational

Colloq Vitae 2020 set-dez; 12(3): 10-15.

Artigo Open Access sob uma licença CC BY-NC-ND (http://creativecommons.org/licenses/by-nc-nd/4.0/). 
Inheritance of Pathologies and Sperm

Epimutations: Generational Toxicology. Sci Rep.

2019; 9(1): 6372.DOI:

https://doi.org/10.1038/s41598-019-42860-0.

10.Turkmen R, Birdane YO, Demirel HH, Kabu M, Ince $S$. Protective effects of resveratrol on biomarkers of oxidative stress, biochemical and histopathological changes induced by sub-chronic oral glyphosate-based herbicide in rats. Toxicol Res (Camb). 2019 ;8(2):238-45. DOI: https://doi.org/10.1039/c8tx00287h. 